\title{
The coherence method using a thin anisotropic elastic plate model
}

\author{
C. J. Swain ${ }^{1}$ and J. F. Kirby \\ Department of Spatial Sciences, Curtin University, WA, Australia
}

Received 6 August 2003; accepted 12 September 2003; published 14 October 2003.

[1] The coherence method, based on the statistical correlation between gravity and topography, has been widely used to estimate the effective elastic thickness of the continental lithosphere and its variations. By far the majority of studies have assumed an isotropic lithosphere, but several papers have inferred anisotropy from differences in the observed coherence (or admittance) functions in different directions. However, none have used an anisotropic model to fit the coherence. We show how the simplest model of elastic anisotropy (an orthotropic thin plate) can be incorporated into both forward and inverse modeling algorithms, using an appropriate approximation in the partial differential equation for the flexure of such a plate. INDEX TERMS: 1236 Geodesy and Gravity: Rheology of the lithosphere and mantle (8160); 8110 Tectonophysics: Continental tectonics-general (0905); 8164 Tectonophysics: Stresses - crust and lithosphere. Citation: Swain, C. J., and J. F. Kirby, The coherence method using a thin anisotropic elastic plate model, Geophys. Res. Lett., 30(19), 2014, doi:10.1029/ 2003GL018350, 2003.

\section{Introduction}

[2] In modern isostatic analysis the lithosphere is often treated as a thin elastic plate, overlying a fluid asthenosphere, and loaded by both surface topography and subsurface density anomalies [Watts, 2001]. The resulting flexure deforms the Moho and can thus be inferred from gravity anomalies, which also reflect the subsurface loads. The mechanical strength of the lithosphere can be quantified by its flexural rigidity $(D)$ or effective elastic thickness $\left(T_{e}\right)$, which can be determined either by modeling the gravity and topography data together, or by using statistical methods, based on the FFT, to estimate the admittance or coherence of the two data sets from their cross-spectra. The utility of $T_{e}$ estimates for regional tectonic studies was highlighted by Lowry and Smith [1995] who showed that the significant controlling factors are temperature, composition and stress, so that $T_{e}$ variations frequently correlate with both tectonic provinces and seismicity.

[3] In Forsyth's [1985] method the flexural rigidity $D$ is estimated by modeling the observed coherence between Bouguer gravity and topography. Most studies have used periodogram spectral estimation, after mirroring the data sets in their edges to remove first order discontinuities. More recently 2-D multitapers have been used for estimating the coherence; Simons et al. [2000] noted that this resulted in much lower $T_{e}$ estimates. In a study similar to

${ }^{1}$ Permanently at 20 Bedwell Crescent, Booragoon, WA 6154, Australia.

Copyright 2003 by the American Geophysical Union. 0094-8276/03/2003GL018350\$05.00 that of Macario et al. [1995], Swain and Kirby [2003] showed that use of multitapers causes a systematic downward bias in $T_{e}$ estimation by Forsyth's method which we suggested could be corrected by means of "calibration curves" based on synthetic model results. We found that the bias increases with increasing multitaper parameters $N W$ (resolution bandwidth) and $K$ (number of tapers along each axis) and with decreasing window size.

[4] Only a few studies of anisotropic $T_{e}$ have been published. Stephenson and Lambeck [1985] divided the 2-D admittance $Q(\boldsymbol{k})$ for central Australia into NS and EW subsets $\left( \pm 45^{\circ}\right)$ prior to azimuthal averaging and found clear evidence for anisotropy in their $Q_{E W}(k)$ and $Q_{N S}(k)$ despite their rather large error estimates due to the use of a single data taper (which was conventional practice at the time). Lowry and Smith [1995] used a somewhat similar technique based on inverting the 1-D functions formed by averaging over subsets of 2-D coherence centered at different azimuths to generate estimates of $T_{e}$ as a function of azimuth for the western U.S. The anisotropy of central Australia was further investigated by Simons et al. [2000] who took advantage of the multitaper method to generate a 2-D coherence function $\gamma^{2}(\boldsymbol{k})$ which is also clearly anisotropic. Simons et al. [2003] used Hermite functions as data tapers for estimating $\gamma^{2}(\boldsymbol{k})$ and its spatial variation; they found that at long wavelengths, corresponding to the transition from high to low coherence, the weak directions are approximately NW-SE in central Australia.

[5] None of the papers cited uses an anisotropic plate model to interpret their data. In the next section we describe such a model and show how to generate synthetic data from it.

\section{An Anisotropic Thin Plate Model}

[6] The differential equation for bending of a thin orthotropic plate is given in several engineering texts [e.g. Szilard, 1974]. Taking the 3 planes of symmetry as the coordinate planes, and using the model of Banks et al. [2001] for a thin plate with both surface $\left(\ell_{T}\right)$ and subsurface $\left(\ell_{B}\right)$ initial loads and overlying an inviscid fluid (density $\rho_{m}$ ) the deflection $w$ is given by:

$$
D_{x} \frac{\partial^{4} w}{\partial x^{4}}+2 H \frac{\partial^{4} w}{\partial x^{2} \partial y^{2}}+D_{y} \frac{\partial^{4} w}{\partial y^{4}}=-\ell_{T}-\ell_{B}-\rho_{m} g w
$$

where $H=1 / 2\left(\sigma_{y} D_{x}+\sigma_{x} D_{y}\right)+2 D_{t}$ for an intrinsically orthotropic material (i.e., one with anisotropic elastic constants), $\sigma$ being Poisson's ratio and $D_{t}$ the torsional rigidity. For such a material it is shown by Szilard [1974] that $\sigma_{x} D_{y}=\sigma_{y} D_{x} \approx \sqrt{ }\left(\sigma_{x} \sigma_{y} D_{x} D_{y}\right)$, and that $H \approx \sqrt{ }\left(D_{x} D_{y}\right)$ so when $D_{x}=D_{y}$ equation (1) reduces to the standard isotropic 2-D biharmonic equation. We have tried using $H=$ 


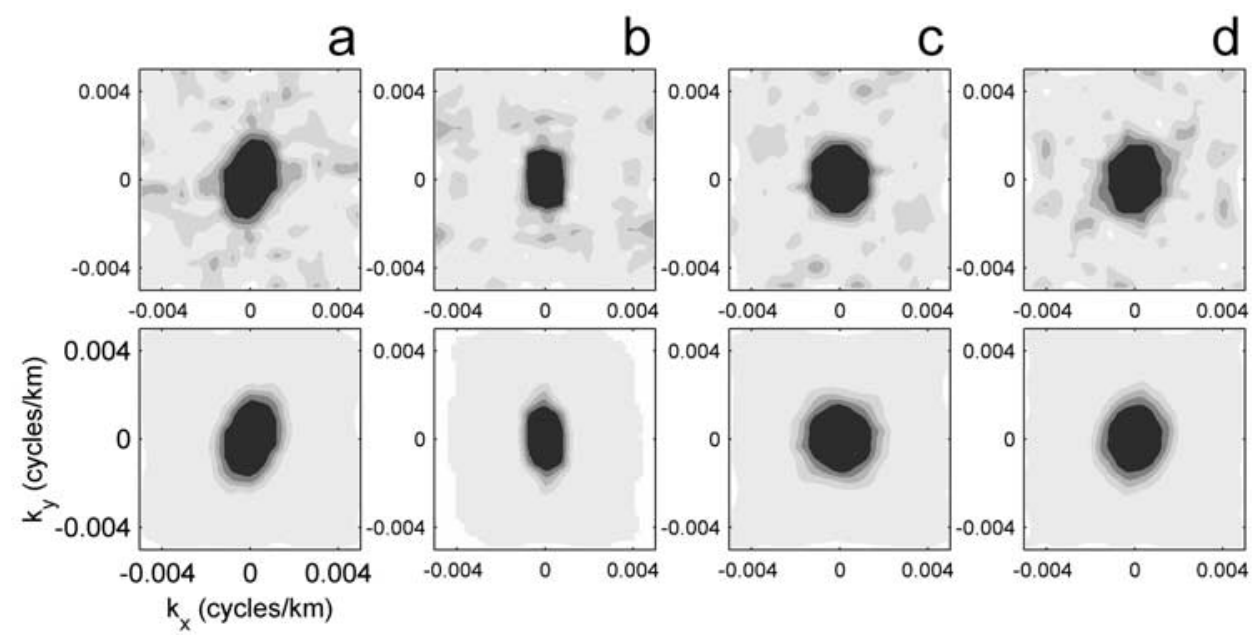

Figure 1. Observed (upper) and best-fitting predicted (lower) multitaper 2-D coherences for 4 of the 100 anisotropic models, each with $T_{x}=100 \mathrm{~km}, T_{y}=50 \mathrm{~km}, \theta=0$, and random fractal surface and subsurface loads. $N W=3$ and $K=3$. Grey scale interval is 0.2 and darkest tone is $>0.8$. The model parameter estimates $\left(\left\langle T_{x}\right\rangle \mathrm{km},\left\langle T_{y}\right\rangle \mathrm{km},\langle\theta\rangle^{\circ}\right)$ are: $(\mathrm{a})(72,35$, 14); (b) $(98,41,-4)$; (c) $(52,41,-54)$; (d) $(59,43,0)$.

$\alpha \sqrt{ }\left(D_{x} D_{y}\right)$ in place of the above approximation and found only small changes to the observed coherence generated from our models for different values of $\alpha$ less than about 3 , so we have assumed $\alpha=1$.

[7] In the wavenumber domain the equation becomes:

$$
\left(D_{x} k_{x}^{4}+2 H k_{x}^{2} k_{y}^{2}+D_{y} k_{y}^{4}+\rho_{m} \mathrm{~g}\right) W=-L_{T}-L_{B}
$$

where $W, L_{T}, L_{B}$ are the Fourier transforms of the plate deflection and loads. Note that with the approximation for $H$ given above, this is the same as equation (16) of Banks et al. [2001] with their $D k^{4}$ replaced by $\left(\sqrt{ } D_{x} k_{x}^{2}+\sqrt{ } D_{y} k_{y}^{2}\right)^{2}$, so calculating the deflection of such an anisotropic plate is hardly more difficult than for the isotropic case. If the direction of anisotropy is not aligned along one of the axes, a rotation of $\left(k_{x}, k_{y}\right)$ through angle $\theta$ is required, i.e. $k_{x}^{\prime}=$ $k_{x} \cos \theta-k_{y} \sin \theta, k_{y}^{\prime}=k_{y} \sin \theta+k_{x} \cos \theta$. The topography can be calculated as the sum of the initial topography $L_{T} /\left(\rho_{0} g\right)$ and the flexure $W$, and the resulting Bouguer gravity anomaly as the sum of the effects of the internal load and Moho deflection [Banks et al., 2001; Equations 17, 18]. Note that in this model $L_{B}$ takes the form of a thin sheet of variable density. In this study we have used random fractal loads [Macario et al., 1995; Swain and Kirby, 2003].

\section{Parameter Estimation}

[8] The essence of Forsyth's [1985] coherence method is to invert the above process and estimate the initial loads from the observed topography and Bouguer gravity assuming a value for $D$, and hence calculate a predicted coherence. $D$ can then be estimated as the value that minimizes the misfit between observed and predicted coherence. For isotropic $D$, coherence estimates and their errors are generally made in 1-D by averaging between annuli. The "load deconvolution" equations, for calculating the loads from the observed gravity/topography for the thin sheet model, were given by Banks et al. [2001], with a correction by Swain and Kirby [2003].
[9] With the anisotropic model described above the only change required to the load deconvolution equations is (as before) to replace $D k^{4}$ by $\left(\sqrt{ } D_{x} k_{x}^{2}+\sqrt{ } D_{y} k_{y}^{2}\right)^{2}$. There are now 3 parameters to be estimated: $D_{x}, D_{y}$ and the azimuth $\theta$. The 2-D coherence estimates that are required were made using multitapers. Jackknifed error estimates [Thomson and Chave, 1991] were used in calculating a misfit $\varepsilon$ [Tarantola, 1987]. We have found it useful to include only the low wavenumbers (e.g., $<3 k_{t}$ where $k_{t}$ is the transition wavenumber). We also weight the observations by $1 / k^{2}$ so that the results are compatible with those that assume an isotropic plate and perform annular averaging with equal increments of $\log k$. We have found that $\varepsilon\left(T_{x}, T_{y}, \theta\right)$ is almost always a very smooth function with a single minimum, so the problem is quasi-linear and parameter estimation by means of an iterative least-squares algorithm works well [Tarantola, 1987, equation 1.100]. Speed of convergence depends on the starting model but usually requires 4 iterations. Although damping is often not required, we include it by means of a priori parameter variances [Tarantola, 1987]; the values we typically use are equivalent to uncertainties of $100 \mathrm{~km}$ in $T_{x}, T_{y}$ and $10^{\circ}$ in $\theta$. This means that $\theta$ is more strongly damped than $T_{x}$ and $T_{y}$ which takes care of the fact that if anisotropy is small then $\theta$ is poorly determined.

[10] It is worth noting that we also tried averaging the coherences within elliptical annular rings. Our motivation for this was the observation that 1-D misfit as a function of $D$ for the isotropic case gives quite narrow minima when calculated by averaging multitaper coherence between annuli, whereas the 2-D misfit, calculated as described above, gives broad minima. However, we found that $\varepsilon\left(D_{x}\right.$, $D_{y}$ ) for a given azimuth then has the form of a long narrow trough, with $D_{x}$ and $D_{y}$ highly correlated, so we abandoned this approach.

\section{Some Model Results}

[11] We present results from a run of 100 models with random fractal loads and $D_{x}=8.9 \times 10^{24} \mathrm{~N} / \mathrm{m}$ 


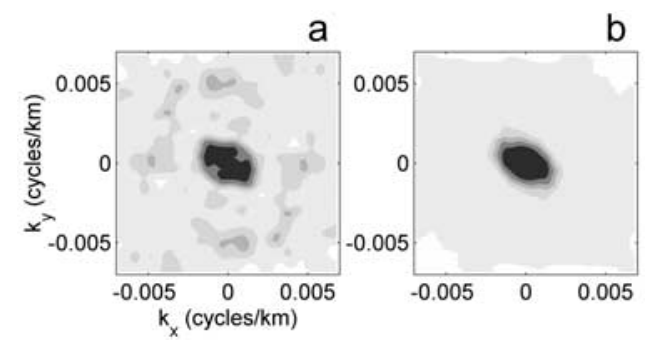

Figure 2. Observed (a) and best-fitting predicted (b) multitaper 2-D coherences for central Australia. $N W=3$, $K=3$. Grey scale interval is 0.2 and darkest tone is $>0.8$. Coherence is predicted by an anisotropic model with $T_{x}=$ $79 \mathrm{~km}, T_{y}=54 \mathrm{~km}, \theta=-50^{\circ}$.

$\left(T_{x}=100 \mathrm{~km}\right), D_{y}=1.1 \times 10^{24} \mathrm{~N} / \mathrm{m}\left(T_{y}=50 \mathrm{~km}\right)$ and $\theta=0$. The surface and subsurface loads were made equal at all wavenumbers $(f=1)$. We assumed $D$ to be related to effective elastic thickness $T_{e}$, Young's modulus $E,\left(10^{11} \mathrm{~Pa}\right)$ and Poisson's ratio $\sigma(0.25)$ by the isotropic formula: $D=$ $E T_{e}^{3} /\left[12\left(1-\sigma^{2}\right)\right]=\sim 8.9 \times 10^{18} T_{e}^{3} \mathrm{Nm}$, with $T_{e}$ in $\mathrm{km}$. We used a window size of $256 \times 256$ with a $10 \mathrm{~km}$ cell size, a resolution bandwidth $N W=3$ and number of tapers $K=3$ for all spectral estimates. [We found that although use of $N W=2$ and $K=2$ [Swain and Kirby, 2003] may be adequate for 1-D coherence with annular averaging, with 2-D coherence larger values are required even for synthetic data.]

[12] Figure 1 shows observed and best-fitting predicted coherences for 4 of the models, selected to illustrate the large variation between results for models with identical elastic parameters, rather than typical results. Since the models only differ in their loads, these results clearly illustrate the important fact that anisotropy of the 2-D coherence can arise from anisotropy of the loading ratio $f(\boldsymbol{k})$ as well as from anisotropy of $D$. Figure 1d shows that the inversion can correctly estimate the anisotropy direction even when the azimuth indicated by the coherence is $>20^{\circ}$ in error.

[13] Histograms of the model parameters for the 100 models look reasonably Gaussian but we have noticed that outliers sometimes occur at $>3$ SDs above $\left\langle T_{x}\right\rangle$, so we prefer to use medians (this is referred to again in the next paragraph). The median flexural rigidity estimates and SDs for all 100 models are: $\left\langle D_{x}\right\rangle=2.8(+1.8-1.1) \times 10^{24} \mathrm{Nm}$, $\left\langle D_{y}\right\rangle=6.6(+4.1-2.5) \times 10^{23} \mathrm{Nm}\left(\left\langle T_{x}\right\rangle=68 \pm 11 \mathrm{~km}\right.$, $\left.\left\langle T_{y}\right\rangle=43 \pm 7 \mathrm{~km}\right)$ and $\langle\theta\rangle=-1 \pm 17^{\circ}$. The results for 2 sets of 100 isotropic models with $T_{e}=100 \mathrm{~km}$ and $50 \mathrm{~km}$ were $\left\langle T_{e}\right\rangle=68 \pm 7 \mathrm{~km}$ and $\left\langle T_{e}\right\rangle=42 \pm 3 \mathrm{~km}$, respectively, indicating a similar bias for isotropic and anisotropic $T_{e}$ estimates made with the same multitaper parameters. This means that we can use an error curve for isotropic models, as in Swain and Kirby [2003], to make approximate corrections for the downward bias.

[14] We have noticed that the outliers referred to above are associated with the $1 / k^{2}$ weighting of the observed coherences in the inversion, particularly where $T_{e}$ is large, and tend not to occur where there is no weighting, or where the weight is an inverse function of a smaller power of $k$. We have also noticed their occurrence when annular averaging is used with equal increments of $\log k$, when the plate is assumed to be isotropic: as noted above, this corresponds to $1 / k^{2}$ weighting. For example, Figure $1 \mathrm{c}$ of Macario et al. [1995] shows several estimates at $\sim 140 \mathrm{~km}$ for a true $T_{e}$ of $80 \mathrm{~km}$. Using the median, rather than the mean, for their data gives $\left\langle T_{e}\right\rangle=86 \mathrm{~km}$ (the mean is given as $95 \pm 29 \mathrm{~km}$ ). This calls into question their conclusion that "there is a strong likelihood of an upward bias in $T_{e}$ estimates if the dimensions of the study area are not adequate to fully resolve the longest wavelengths". We think that a more justified conclusion would be that small window sizes, relative to the flexural wavelength, give an increased likelihood of spuriously high $T_{e}$ estimates. It would probably be better to use a weighting that reduces the likelihood of such outliers, but we use the $1 / k^{2}$ weighting here purely for compatibility between isotropic and (average) anisotropic results.

\section{Anisotropic Elastic Plate Model for Central Australia}

[15] We used this model to fit the coherence between Bouguer gravity and topography data from a $2200 \times$ $2200 \mathrm{~km}$ area of central Australia, centred at $23.5^{\circ} \mathrm{S}$, $133^{\circ} \mathrm{E}$. This is the same data set studied by Swain and Kirby [2003]. Figure 2 shows the observed and best-fitting predicted coherences. The parameter estimates for this model are $D_{x}=4.4 \times 10^{24} \mathrm{Nm}, D_{y}=1.4 \times 10^{24} \mathrm{Nm}\left(T_{x}=\right.$ $\left.79 \mathrm{~km}, T_{y}=54 \mathrm{~km}\right)$ and $\theta=-50^{\circ}$, i.e. the azimuth of the weak direction is $-50^{\circ} \mathrm{N}$. This direction is similar to the "long-wavelength weak directions" for 3 points in central Australia shown in Figure 12a of Simons et al. [2003].

[16] In order to correct our $T_{e}$ estimates for multitaper bias [Swain and Kirby, 2003], we have constructed error (or calibration) curves for the multitaper parameters $N W=$ $3, K=3$ and a window size of $2200 \times 2200 \mathrm{~km}$. Figure 3 shows the results for isotropic models with low $\mathrm{S} / \mathrm{N}$ ratio, as defined in Swain and Kirby [2003] and appropriate for central Australia. Comparison with Figure 2 of Swain and Kirby [2003] clearly shows the increase in bias resulting from the larger resolution bandwidth $(N W)$ and number of

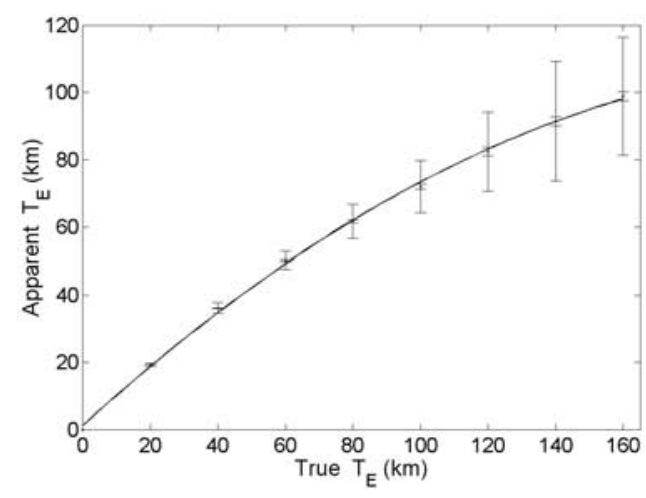

Figure 3. Error curves for multitaper coherence $T_{\mathrm{e}}$ estimates (with $K=3, N W=3$ ) for a square window of width $2200 \mathrm{~km}$. Each point is the result of applying the $T_{\mathrm{e}}$ estimation program to a set of 100 pairs of synthetic topography and gravity grids. The curve is a second degree polynomial. The error bars show both standard errors and standard deviations. 
tapers $(K)$. From this curve we estimate that the apparent $T_{e}$ values of 79 and $54 \mathrm{~km}$ correspond to true values of about $T_{x}=120 \mathrm{~km}, T_{y}=70 \mathrm{~km}$. To make error estimates we inverted 100 data sets created from an anisotropic model with these $T_{x}$ and $T_{y}$ values, $\theta=0$, and with low $\mathrm{S} / \mathrm{N}$ ratio. The results were $\left\langle T_{x}\right\rangle=80 \pm 17 \mathrm{~km},\left\langle T_{y}\right\rangle=55 \pm 11 \mathrm{~km}$, $\langle\theta\rangle=0 \pm 23^{\circ}$. From these results we can estimate the corrected values as $T_{x}=120-30 /+40 \mathrm{~km}, T_{y}=70 \pm 15 \mathrm{~km}$ (with errors estimated by reading off from Figure 3 the "True $T_{e}$ " values corresponding to the errors given for each uncorrected estimate). The corrected flexural rigidities for central Australia are $D_{x}=1.5(+2.1-0.9) \times 10^{25} \mathrm{Nm}, D_{y}=$ $3.0(+2.5-1.5) \times 10^{24} \mathrm{Nm}$, and the azimuth of the weak direction is $-50^{\circ} \mathrm{N} \pm 23^{\circ}$.

\section{Comparison With the Isotropic Result for Australia}

[17] The isotropic $T_{e}$ estimate for central Australia given in Swain and Kirby [2003] was $115 \pm 25 \mathrm{~km}$ (corrected for multitaper bias). We would expect the average of the $T_{x}, T_{y}$ estimates for an anisotropic model (or perhaps their geometric mean) to approximate this, and the fact that it is somewhat lower $(95 \mathrm{~km})$, though within the error estimates, requires comment.

[18] Because the multitaper parameters were different in the two studies, we obtained an isotropic estimate using the multitaper parameters $N W=3, K=3$ and corrected this using Figure 3, obtaining $T_{e}=100 \pm 20 \mathrm{~km}$. We consider that the difference between this value and the anisotropic average does not indicate any systematic bias.

[19] The difference between the two isotropic estimates for the same data set $(115 \mathrm{~km}$ with $N W=2, K=2 ; 100 \mathrm{~km}$ with $N W=3, K=3$ ) is probably due to differences in the behavior of the estimation procedure for the synthetic and real data. One possible contributing factor could be that the azimuthally averaged maximum observed coherence for the Australian data (0.81) is somewhat lower than for synthetic data sets $(>0.95$ in most cases $)$.

\section{Discussion and Conclusions}

[20] We have shown that it is easy to incorporate anisotropy into both forward wavenumber-domain modeling and Forsyth's [1985] inverse method by means of an intrinsically orthotropic thin plate model. The new method appears to work well and has the important advantages, compared to non-model-based methods, of fitting the model to all the observed data (2-D coherences) and of accounting for the effects of anisotropic loading. However, it is perhaps necessary to ask whether such a model can approximate the mechanical behaviour of the lithosphere. Simons et al. [2000] noted a number of possible mechanisms to explain azimuthal variations in $T_{e}$ including: (a) stress; (b) anisotropic variations of Moho depth and (c) its temperature; (d) intrinsic anisotropy of mantle materials; (e) parallel faulting; (f) alignment of weak zones in the crust. However, Lowry and Smith [1995] suggested that lithospheric weakening by regional horizontal stress is the most likely cause. We infer that mechanisms (b)-(f) can all be approximated by an intrinsically anisotropic plate. The case of stress-induced anisotropy of $T_{e}$ is more difficult as the azimuthal pattern of $T_{e}$ variation can be bilobate or cloverleaf as well as elliptical, depending on the stress pattern [Lowry and Smith, 1995]. This is because $T_{e}$ varies non-linearly with stress and the latter varies with azimuth $\theta$ as $\cos ^{2} \theta$. However, we have converted such azimuthal $T_{e}$ variations to coherence transition wavenumbers, which are approximately elliptical, so the model is probably adequate in this case as well.

[21] As well as the central elliptical high, the coherence map (Figure 2a) shows anomalous high coherence on the $k_{y}$-axis at a wavelength of $\sim 200 \mathrm{~km}$. The admittance map shows a very similar central elliptical anomaly (with values of about $-0.12 \mathrm{mGal} / \mathrm{m}$ ) and also a similar anomaly on the $k_{y}$-axis which is so strong that it is still significant after azimuthal averaging [Swain and Kirby, 2003, Figure 4]. It results from the negative correlation that exists at these (and shorter) wavelengths between the major EW gravity anomalies of central Australia and the topography, due to NS compressional tectonics.

[22] Although our weak direction of $T_{e}$ anisotropy, $-50^{\circ} \mathrm{N} \pm 23^{\circ}$, is inconsistent with this NS compression, present-day stress directions within our area [Hillis et al., 1998] vary from $10^{\circ}-20^{\circ} \mathrm{N}$ in the Amadeus Basin to $70^{\circ} \mathrm{N}$ just $200 \mathrm{~km}$ to its south, and approximately EW a few hundred $\mathrm{km}$ to the east and west. Our $T_{e}$ anisotropy direction is also at odds with the predominantly EW directions of major faults, possible weak zones in the crust and of inferred Moho topography. Simons et al. [2003] argue that the direction of $T_{e}$ anisotropy should be perpendicular to the fast axis of seismic anisotropy. In both their anisotropic velocity model and that of Debayle and Kennett [2000], the fast axis varies spatially very rapidly at depths $<150 \mathrm{~km}$, although it is more uniform, with direction $0^{\circ}-30^{\circ} \mathrm{N}$, at depths $>150 \mathrm{~km}$. We conclude that although is impossible to correlate our direction of $T_{e}$ anisotropy to the regional stress field or to velocity anisotropy in the upper lithosphere, it could represent an average of either of these vector fields over our large data window.

[23] Acknowledgments. We thank Tony Lowry for commenting on an earlier version of the paper. The Australian Research Council supported this work with grant no. DP0211877.

\section{References}

Banks, R. J., S. C. Francis, and R. G. Hipkin, Effects of loads in the upper crust on estimates of the elastic thickness of the lithosphere, Geophys. J. Int., 145, 291-299, 2001.

Debayle, E., and B. L. N. Kennett, The Australian continental upper mantle: Structure and deformation inferred from surface waves, J. Geophys. Res., $105,25,423-25,450,2000$.

Forsyth, D. W., Subsurface loading and estimates of the flexural rigidity of continental lithosphere, J. Geophys. Res., 90, 12,623-12,632, 1985.

Hillis, R. R., J. J. Meyer, and S. D. Reynolds, The Australian stress map, Explor. Geophys., 29, 420-427, 1998.

Lowry, A. R., and R. B. Smith, Strength and rheology of the western U. S. Cordillera, J. Geophys. Res., 100, 17,947-17,963, 1995.

Macario, A., A. Malinverno, and W. F. Haxby, On the robustness of elastic thickness estimates obtained using the coherence method, J. Geophys. Res., 100, 15,163-15,172, 1995.

Simons, F. J., R. D. van der Hilst, and M. T. Zuber, Spatio-spectral localization of isostatic coherence anisotropy in Australia and its relation to seismic anisotropy: Implications for lithospheric deformation, J. Geophys. Res., 108(B5), 2250, doi:10.1029/2001JB000704, 2003.

Simons, F. J., M. T. Zuber, and J. Korenaga, Isostatic response of the Australian lithosphere: Estimation of effective elastic thickness and anisotropy using multitaper spectral analysis, J. Geophys. Res., 105, $19,163-19,184,2000$. 
Stephenson, R., and K. Lambeck, Isostatic response of the lithosphere with in-plane stress: Application to Central Australia, J. Geophys. Res., 90, $8581-8588,1985$.

Swain, C. J., and J. Kirby, The effect of 'noise' on estimates of the elastic thickness of the continental lithosphere by the coherence method, Geophys. Res. Lett., 30(11), 1574, doi:10.1029/2003GL017070, 2003.

Szilard, R., Theory and Analysis of Plates, Prentice-Hall, Englewood Cliffs, N. J., 1974.

Tarantola, A., Inverse Problem Theory, Elsevier, Amsterdam, 1987.

Thomson, D. J., and A. D. Chave, Jackknifed error estimates for spectra, coherences, and transfer functions, in Advances in Spectrum Analysis and
Array Processing, edited by S. Haykin, 1(2), 58-113, Prentice Hall, Englewood Cliffs, N. J., 1991.

Watts, A. B., Isostasy and Flexure of the Lithosphere, Cambridge Univ. Press, 2001.

J. F. Kirby, Department of Spatial Sciences, Curtin University, WA, Australia.

C. J. Swain, 20 Bedwell Crescent, Booragoon, WA 6154, Australia. (c_swain@wt.com.au) 